Check for updates

Cite this: RSC Adv., 2017, 7, 32737

\title{
2D material integrated macroporous electrodes for Li-ion batteries†
}

\author{
Hemtej Gullapalli, (D)* Kaushik Kalaga, Soumya Vinod, Marco-Tulio F. Rodrigues, \\ Antony Georget and Pulickel M. Ajayan
}

Three-dimensionally structured architectures are known to improve the performance of electrodes used in $\mathrm{Li}$ ion battery systems. In addition, integration of select 2D materials into 3D structures, for enhancing both electrical conductivity and electrochemical activity, will prove advantageous. Here a scalable one-step chemical vapor deposition technique is demonstrated for the controlled etching and simultaneous graphene growth on stainless steel substrates resulting in a 3D micro-mesh architecture that is ideal for high rate/high capacity electrodes; the graphene coated 3D stainless steel current collector is used with an $\mathrm{MoS}_{2}$ electrode material for demonstrating high stability and rate capacity in Li-ion batteries.

Received 12th May 2017

Accepted 19th June 2017

DOI: 10.1039/c7ra05354a

rsc.li/rsc-advances penetration of the carbon precursor into the 3D network and/or collapse of the $3 \mathrm{D}$ structure at high deposition temperatures.

In the context of thin film and miniaturized energy storage, as the size of the device shrinks, the equivalent resistances increases exponentially, limiting the performance. In order to overcome this, proficient packing of electrode material is required, which is a challenge in a planar configuration. Charge transfer to the bulk of the electrode in a high aspect ratio format can only be achieved by employing a 3D configuration for the current collector. For this, the current collector would need to withstand the rigorous processing techniques used for homogenous deposition of most electrode materials. Moreover, the primary attribute of a current collector is to have an electrochemical inertness across the voltage of device operation and a minimal surface charge resistance facilitating good electron transport from the electrode. Thus towards realizing such configuration, in this report, we demonstrate a one-step CVD process to transform thermally and chemically stable planar stainless steel metallic substrates into micron-sized mesh like 3D architectures with conformal graphene covering on the surface which aids in increasing electrochemical stability and electron transfer.

Such current collectors are clearly advantageous for use with high capacity anode materials, which are plagued by low conductivity. Ceramic materials like $\mathrm{MnO}_{2},{ }^{22,23} \mathrm{MoS}_{2}{ }^{24}$ and others have demonstrated excellent capacity (up to $1290 \mathrm{~mA} \mathrm{~h}$ $\mathrm{g}^{-1}$ for $\mathrm{MoS}_{2}$ ) compared to pristine carbon anodes. Several methodologies such as structural tuning and incorporating these materials into carbon host structures has been shown to alleviate the conductivity issue. ${ }^{25-28}$ However, the net volumetric capacity was compromised in such approaches. Of these ceramic anodes, 2D transition metal di-chalcogenides (TMD's) are particularly interesting compared to oxide counterparts due to better first cycle efficiencies and smaller volume
Department of Materials Science and Nano Engineering, Rice University, Houston, TX, USA.E-mail: hemtej@rice.edu

$\dagger$ Electronic supplementary information (ESI) available. See DOI: 10.1039/c7ra05354a

\$ Institute of Physical Chemistry, Friedrich Schiller University Jena, Jena, Germany. 
changes. ${ }^{29,30,36,37}$ In this report, we validate the efficacy of 3D graphene covered stainless-steel current collectors by incorporating $2 \mathrm{D} \mathrm{MoS}_{2}$ into the pores using a controlled electrodeposition technique.

\section{Experimental procedure}

\section{Fabrication of graphene covered 3D stainless steel}

Chemical vapor deposition was done using a split tube furnace fitted with a quartz tube and equipped with a vacuum evacuation feature. The setup has facility for a liquid vapor source connected by a tabulated flask. The flask has a shutoff valve and can be transferred into a glovebox to be filled with inert precursors. Perfluorohexane (aka- $\mathrm{C}_{6} \mathrm{~F}_{14}$ or tetradecafluorohexane, hereby called $\mathrm{PFH}$ ) (99\% purity and sealed under argon, from Sigma Aldrich) was used as a carbon precursor. 304grade stainless steel foil (Alfa Acer) was used as the substrate. The substrate-loaded tube was first evacuated and heated to $950{ }^{\circ} \mathrm{C}$ while flowing $\mathrm{Ar} / 15 \% \mathrm{H}_{2}$ and maintaining the pressure at 10 Torr. Once the desired temperature has reached, $\mathrm{H}_{2}$ flow was stopped and the tube was flushed with pure argon for a moment and then turned off. Vapors of PFH were passed for 10 to 12 minutes, while controlling the inlet flow to maintain the pressure at 600 mTorr. The furnace was then cooled down with the flow of PFH continued until the temperature reached $800{ }^{\circ} \mathrm{C}$, and later with a purge of pure Ar flow. The duration of $\mathrm{PFH}$ exposure defines the degree of etch while the rate of cooling dictates the number of graphene layers formed on the surface.

\section{Electro deposition of $\operatorname{MOS}_{2}$}

$\mathrm{MoS}_{2}$ was deposited from a solution of $0.5 \mathrm{~g} \mathrm{~L}^{-1}$ ammonium tetrathiomolybdate $\left(\left(\mathrm{NH}_{4}\right)_{2} \mathrm{MoS}_{4}\right), 3 \mathrm{~g} \mathrm{~L}^{-1}$ ammonium chloride $\left(\mathrm{NH}_{4} \mathrm{Cl}\right)$ and $10.5 \mathrm{~g} \mathrm{~L}^{-1}$ potassium chloride $(\mathrm{KCl})$ in formamide as reported in previous studies. ${ }^{31}$ In the three-electrode setup, the graphene covered stainless steel substrate was used as working electrode, $\mathrm{Ag} / \mathrm{AgCl}$ as reference electrode and platinum wire as counter electrode. Pulsed electrodeposition was carried out at $-0.6 \mathrm{~V}$ (vs. $\mathrm{Ag} / \mathrm{AgCl})$ using potentiostat/galvanostat (AUTOLAB PGSTAT $302 \mathrm{~N}$ ECOCHEMIE) for 5 minutes with ON/OFF time of $10 \mathrm{~s} / 10 \mathrm{~s}$ to ensure uniform deposition on the $3 \mathrm{D}$ structure. The electrolyte solution was maintained at $60{ }^{\circ} \mathrm{C}$ and under constant stirring during the deposition. The substrate after $\mathrm{MoS}_{2}$ deposition was rinsed using DI water and annealed at $450{ }^{\circ} \mathrm{C}$ for 4 hours under Ar atmosphere. Mass loading of $\mathrm{MoS}_{2}$ is measured to be approximately $2 \mathrm{mg} \mathrm{cm}{ }^{-2}$.

\section{Structural characterization}

Morphological analysis of graphene covered 3D stainless steel substrates and $\mathrm{MoS}_{2}$ electrodeposited 3D structures was carried out using scanning electron microscope (FEI, Environmental SEM). Cross-sectional morphology was analyzed by cooling the sample in liquid nitrogen and then shear cracking it. Vibrational properties of the graphene and $\mathrm{MoS}_{2}$ were analyzed using Renishaw Raman spectrometer with a $514.5 \mathrm{~nm}$ wavelength laser. X-ray photoelectron spectroscopy (XPS) studies were carried out with a spectrophotometer (PHI Quantera SXM) using the monochromatic $\mathrm{Al} \mathrm{K} \alpha$ radiation $(1486.6 \mathrm{eV})$.

\section{Electrochemical characterization}

Electrochemical measurements were performed by assembling the samples in a CR2032 type coin cell packaging. For the halfcell measurements, an electrochemical test cell was assembled in Ar-filled glove box using the fabricated 3D structures as working electrode, lithium metal foil as the counter/reference electrode and $1 \mathrm{M}$ solution of $\mathrm{LiPF}_{6}$ in $1: 1(\mathrm{v} / \mathrm{v})$ mixture of ethylene carbonate (EC) and dimethyl carbonate (DMC). Charge discharge cycling tests were conducted using ARBIN Instruments Battery Analyzer while cyclic voltammetry measurements were carried out using a potentiostat/galvanostat (AUTOLAB PGSTAT 302 N ECOCHEMIE).

\section{Results and discussion}

Chemical vapor deposition serves as an appropriate tool to synthesize high quality 3D graphene structures essential for high power LIBs. A schematic of the growth process of 3D graphene on stainless steel shown in Fig. 1(a) is a representation of the etching and subsequent nucleation of graphene on the surface. PFH molecules undergo pyrolysis at the high temperature forming highly reactive fluoride ions and carbon atoms. Reaction of metal atoms in stainless steel with the fluoride ions result in metal fluoride which is carried away by the flowing gas assisted by vacuum suction. New energetic surfaces thus

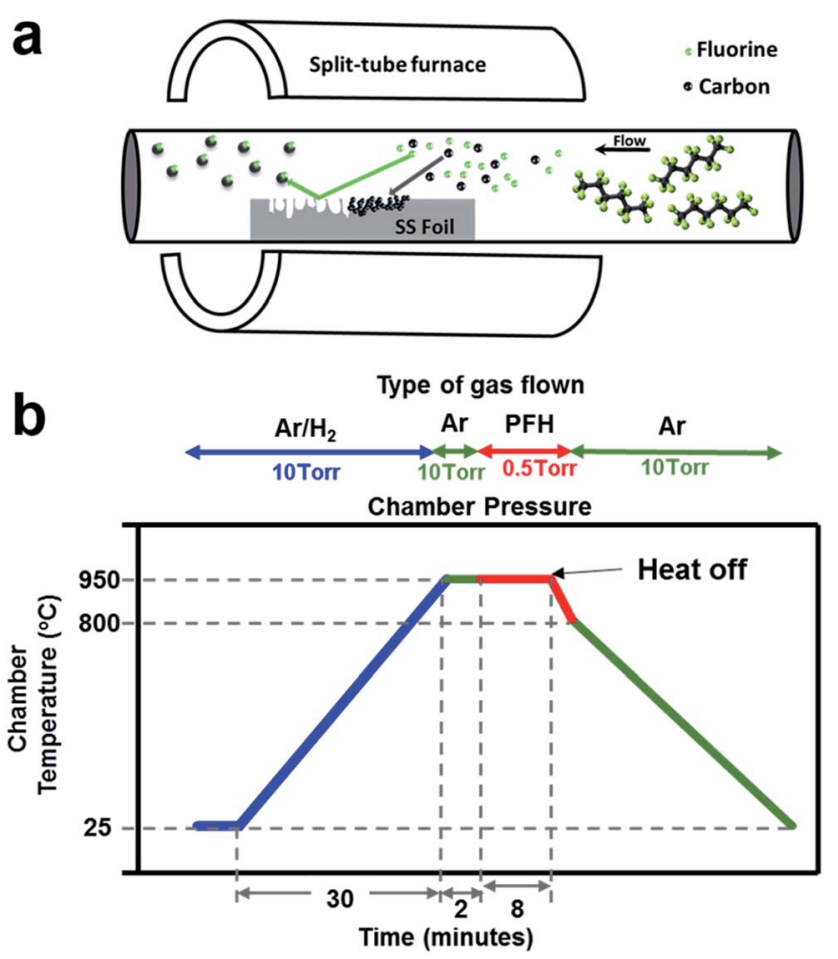

Fig. 1 (a) Schematic depiction of the CVD process for etching the surface of stainless steel while simultaneously forming graphene; (b) process conditions employed for the synthesis. 

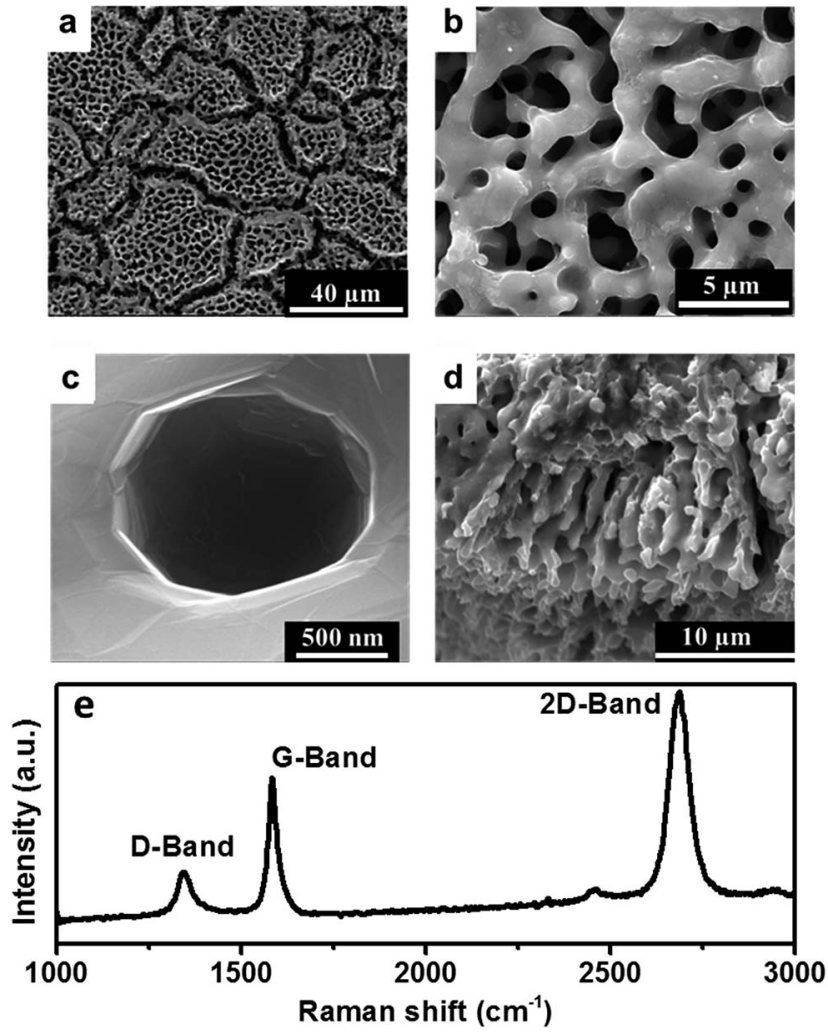

Fig. $2(a-c)$ Scanning electron micrographs of the surface of graphene covered 3D stainless steel substrates at various magnifications; (d) cross-sectional image of the sample, depicting the 3D architecture until a depth of $\sim 10 \mu \mathrm{m}$ from the surface; (e) Raman spectrum of the substrate, confirming 2-3 layered graphene.

formed favor graphene growth through a process of carbon dissolution and subsequent perspiration as understood from a previous study. ${ }^{15}$ The quality of stainless steel, choice of apt precursors and a high degree of process control is necessary for high growth efficiencies. 304-grade austenitic stainless steel foil substrate with limited carbon solubility serves as the best substrate for uniform nucleation of graphene all over the surface. Temperature profile with heating and cooling rates and the dwell time are detrimental for the process and the parameters employed here are shown schematically in Fig. 1(b). Complete purging of the tube with inert (Ar) gas between the reducing $\mathrm{H}_{2}$ gas and the subsequent fluorinated precursor is essential to avoid the formation of any HF species. While the precursor exposure time defines the extent of porosity, an exposure time of 8 to 12 minutes is deemed optimum in this study. The number of graphene layers formed is however not proportionate to the exposure time and is dependent on the cooling rate as reported in our previous study of graphene growth on stainless steel ${ }^{\mathbf{1 5}}$ and the justification holds good here.

Morphological characterization of the obtained graphene films on stainless steel surfaces indicate preferential etching of fluoride ions along the grain boundaries resulting in formation of ridges (Fig. 2(a)). Bulk of the grain transforms into a highly porous 3D foam like network (Fig. 2(b)) with a thin layer of graphene coating. Deeper examinations of the pores shown in Fig. 2(c) reveals the
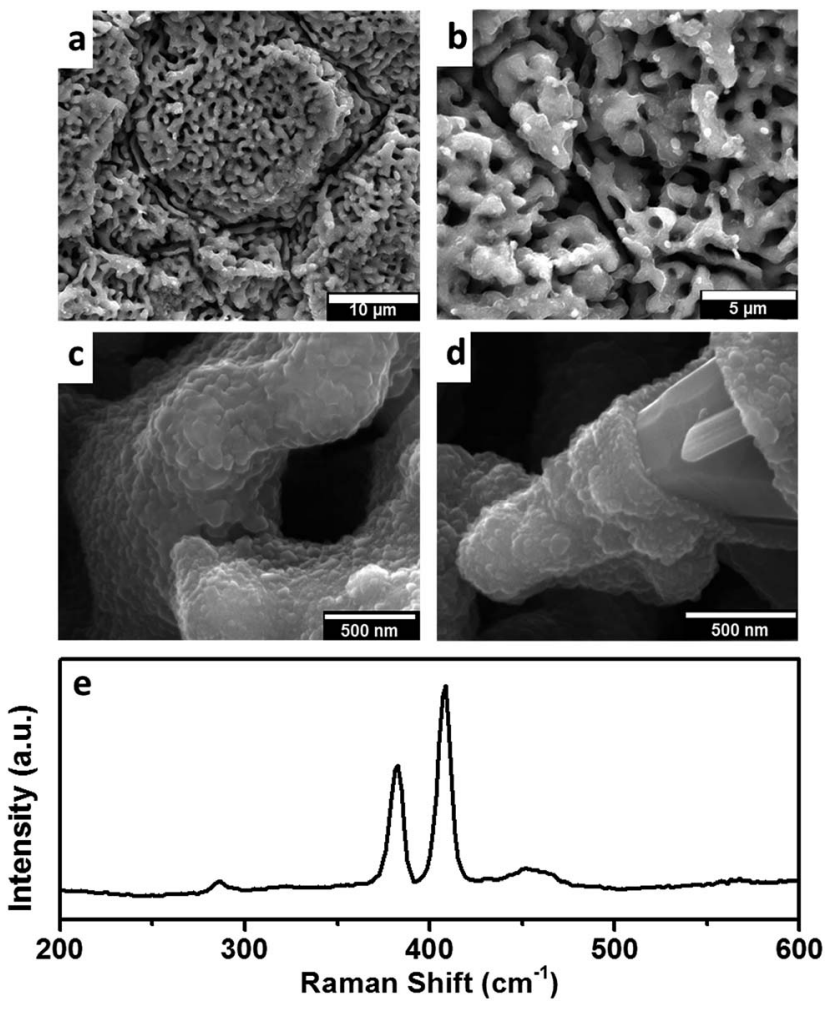

Fig. $3(\mathrm{a}-\mathrm{c})$ Scanning electron micrographs of $\mathrm{MoS}_{2}$ covered 3D stainless steel-graphene structures. A highly conformal coating on the surface is achieved by electrodeposition technique. The porous nature of the current collector is preserved with no clogging or large particulates; (d) an isolated defect in the electrodeposition, shown to highlight the thickness of the $\mathrm{MoS}_{2}$ layer; (e) Raman spectrum of the structure wherein the bands correspond to $\mathrm{MoS}_{2}$.

high extent of conformity of the graphene layer and pore sizes up to $1 \mu \mathrm{m}$. The depth of penetration of the etching process is limited to $10 \mu \mathrm{m}$ from the surface as observed from the cross-sectional image in Fig. 2(d) implying that the etching is a surface phenomenon. This, however, is comparable to thickness of bulk conventional LIB electrodes fabricated using slurry coating methods with the advantage of a 3D structure facilitating cycling at higher rates. Raman spectrum (Fig. 2(e)) of the carbon layer reflects the profile for 2 to 3 layered graphene with the peaks of $\mathrm{G}$ and $2 \mathrm{D}$ bands at $\sim 1675 \mathrm{~cm}^{-1}$ and $\sim 2700 \mathrm{~cm}^{-1}$ respectively having a $\mathrm{G}: \mathrm{D}$ area ratio of 0.3 . A low intensity defect induced $\mathrm{D}$ peak at $\sim 1275 \mathrm{~cm}^{-1}$ reflects the disorder introduced in the graphene lattice due to the curvature of the pores. Elemental analysis using XPS (ESI, Fig. S1 $\dagger$ ) revealed no additional elements other than carbon, indicating purity of the graphene lattice. Though PFH has been used to etch the surface of stainless steel, no residual fluorine was observed in the resultant structure. Elemental XPS analysis of the carbon atoms in the sample (ESI, Fig. S1(b) †) revealed a peak which can be deconvoluted into two constituents, a high intensity peak at $284 \mathrm{eV}$ corresponding to $\mathrm{sp}^{2}$ carbon and an low intensity peak at $285 \mathrm{eV}$ corresponding to $\mathrm{sp}^{3}$ carbons which are a result of the curvature of graphene on the $3 \mathrm{D}$ structure. ${ }^{32}$ Absence of a peak above $286 \mathrm{eV}$ confirms the nonexistence of $\mathrm{C}-\mathrm{F}$ and $\mathrm{C}-\mathrm{O}$ bonds in the structure. ${ }^{33}$ 
Graphite is the most widely used anode in LIBs owing to its high capacity and low lithiation potentials. A preliminary measurement in this direction targeted towards testing the 3D graphene structures (ESI, Fig. S2(a) $\dagger$ ) reveals extraordinary rate capabilities with a high capacity of $20 \mu \mathrm{A} \mathrm{h} \mathrm{cm} \mathrm{cm}^{-2}$ at a current density $64 \mu \mathrm{A} \mathrm{cm}^{-2}$ as compared to $\sim 35 \mu \mathrm{A} \mathrm{h} \mathrm{cm} \mathrm{cm}^{-2}$ at a current density of $2 \mu \mathrm{A} \mathrm{h} \mathrm{cm} ~^{-2}$ for its $2 \mathrm{D}$ counterpart. However, large reversible losses in graphite due to the formation of dynamic solid electrolyte interphase (SEI) plague the battery industry. An additional plateau at $\sim 0.7 \mathrm{~V}$ in the charge discharge profile (ESI, Fig. S2(b) $\dagger$ ) is an indicative of the SEI formation hindering the cyclic stability of the graphene layers.

The evidence of low density of defects from the Raman spectrum indicates the quality of graphene, with its high electronic conductivities turning it into a promising material as a universal current collector for many electrochemically active materials. Recent interest in exploring layered transition metal di-chalcogenide structures as anodes for LIBs motivated electro deposition of $\mathrm{MoS}_{2}$ onto the graphene layer. Parameters optimized from previously reported protocol ${ }^{31}$ resulted in uniform homogenous deposit through the entire region of the grain (Fig. 3(a) and (b)) retaining significant pore volume to accommodate volume changes during cycling. The deposition is controlled to form a continuous and conformal coating, as seen from high magnification SEM images (Fig. 3(c)). A rare defect shown in Fig. 3(d) highlights the compact nature and thickness of the $\mathrm{MoS}_{2}$ layer. With the absence of large pores or pinholes, and the surface is free from deposition of undesired byproducts, which could otherwise clog the pores restricting access to the electrolyte to the interior of the structure. This is a natural consequence of the quality and conformity of the underlying graphene layers, which reduces voltage fluctuations that could lead to the formation of amorphous phases. The distinct and characteristic Raman bands at 390 and $410 \mathrm{~cm}^{-1}$ (Fig. 3(e)) and the absence of additional peaks in the XPS spectra (ESI, Fig. S3 $\uparrow$ ), further confirms the presence of the $\mathrm{MoS}_{2}$ phase.

Having a layered structure, high theoretical capacity and low lithium intercalation potential $(0.8 \mathrm{~V})$ of $\mathrm{MoS}_{2}$ makes it a promising candidate as anode for LIBs. However, low electronic conductivities of $2 \mathrm{D}$ macro particulates in the electrodes impose a barrier towards commercialization. Electrodeposition of nanoparticles of $\mathrm{MoS}_{2}$ onto 3D current collectors attempts to solve the inherent problems of conductivity by reduction in particle sizes and enhancement in effective electrode/electrolyte interface area for better cyclability at high current rates. Cyclic voltammetry scans (Fig. 4(a)) between 0.1 to $3 \mathrm{~V}$ indicate two
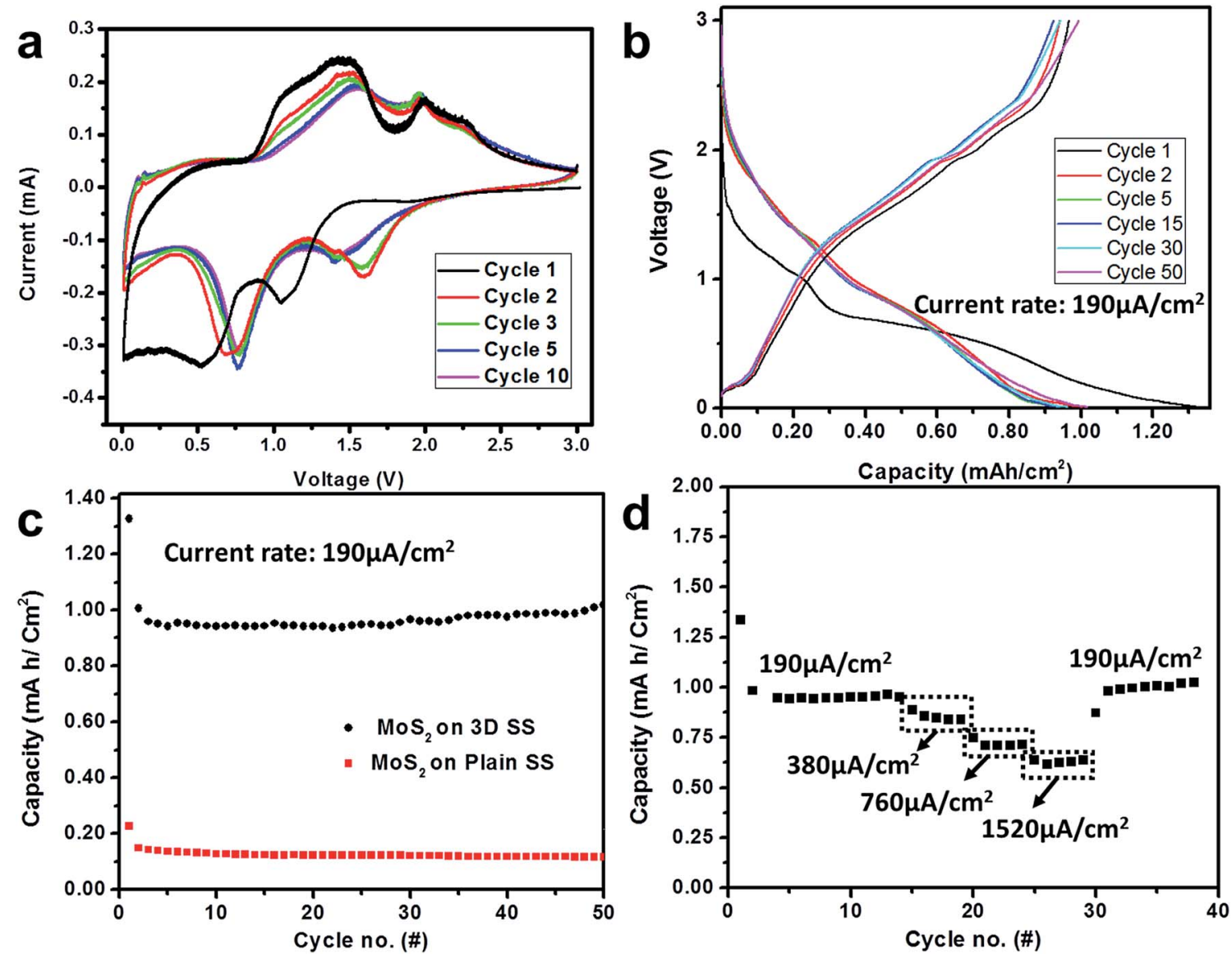

Fig. 4 (a) Cyclic voltammograms of the 3D-MoS 2 electrode in a lithium half-cell. The first cycle involves transformation of MoS 2 from $2 \mathrm{H}$ to $1 \mathrm{~T}$ phase; (b) charge discharge profiles of the cell; (c) cyclic stability studies of the electrode. In comparison with 2D planar substrates, a 5-fold increase in the capacity was observed for the 3D architecture; (d) rate capability testing of the electrode. It can withstand high current rates, as evident from the retention of nominal capacity after returning to lower current rates. 
peaks at $1.1 \mathrm{~V}$ and $0.6 \mathrm{~V}$ during the first discharge, corresponding to lithium insertion into $\mathrm{MoS}_{2}$ and phase change to form $1 \mathrm{~T}-\mathrm{Li}_{x} \mathrm{MoS}_{2}$ and the later dissociation of $\mathrm{MoS}_{2}$ into metallic Mo and $\mathrm{Li}_{2} \mathrm{~S}$ respectively. ${ }^{34,35}$ Subsequent scans demonstrate recurring cathodic peaks at around $0.8 \mathrm{~V}$ and $1.7 \mathrm{~V}$ with corresponding anodic peaks at $1.5 \mathrm{~V}$ and $2 \mathrm{~V}$ respectively, which are characteristic of $\mathrm{MoS}_{2}$ attributed to reversible conversion of $\mathrm{Li}_{2} \mathrm{~S}$ and Mo particles to $\mathrm{Li}_{x} \mathrm{MoS}_{2}$ and the subsequent de-lithiation into $\mathrm{MoS}_{2}$. Consistent occurrence of peaks at the same position over 3 scans indicate complete reversibility of the lithiation and de-lithiation processes with good $\mathrm{MoS}_{2}$-graphene interface and thus effective electron conduction through the current collector. Graphene being only few layers thick, has minimal contribution to the active mass while $\mathrm{MoS}_{2}$ being a denser coating contributes to the gravimetric capacity. The CV profiles observed in Fig. 4(a) confirms such electrochemical activity as there is minimal contribution at voltages $<0.5$ where graphitic carbon is mostly reactive towards lithium ions. Galvanostatic charge discharge profiles (Fig. 4(b)) present a sloping profile of lithiation and de-lithiation with the high over potential characteristic of conversion anode materials. The additional plateau in the first discharge indicating SEI layer formation and does not affect the performance of the electrode substantially, except for a $0.3 \mathrm{~mA} \mathrm{~h} \mathrm{~cm}^{-2}$ loss in capacity. A high capacity of $1.0 \mathrm{~mA} \mathrm{~h} \mathrm{~cm}{ }^{-2}$ and a retention of $\sim 93 \%$ at the end of 50 cycles with a current density of $190 \mu \mathrm{A} \mathrm{cm}^{-2}$ support the hypothesis of good cyclic stability of nanoparticles in combination with 3D architecture as compared to $0.15 \mathrm{~mA} \mathrm{~h} \mathrm{~cm}{ }^{-2}$ for electrodeposited $\mathrm{MoS}_{2}$ electrode onto 2D graphene current collector (Fig. 4(c)).

The 3D $\mathrm{MoS}_{2}$ electrodes exhibit extreme resilience to higher current rates with the ability to store capacity of $0.5 \mathrm{~mA} \mathrm{~h} \mathrm{~cm} \mathrm{~cm}^{-2}$ without any capacity fade over cycling when the current density is increased 8 folds to $1520 \mu \mathrm{A} \mathrm{h} \mathrm{cm}{ }^{-2}$. A complete regain of the nominal capacity was observed when the cell was cycled again at lower current rates (Fig. 4(d)). This implies excellent structural retention of the electrode and thus the robustness of the electrode-current collector combination to tolerate large current fluctuations.

\section{Conclusions}

Using chemical vapor deposition technique, a synthesis process has been developed to etch the surface of stainless steel foils while simultaneously coating it with few-layered graphene. The process has a control on the quality of the deposited graphene, limiting it to 2 to 3 layers with minimal defects and contamination. Having a 3D porous architecture combined with a highly conductive and inert surface coating that does not gravimetrically load the structure, makes the etched stainless steel-graphene an ideal candidate for current collectors in high capacity/high rate electrochemical devices. The efficacy of the structure to support electrodes under high current rates over prolonged cycling is demonstrated by depositing $\mathrm{MoS}_{2}$ into the pores. The 3D architecture had resulted in nearly 4-fold improvement in discharge capacity as compared to a $2 \mathrm{D}$ architecture. Conformal deposition of $\mathrm{MoS}_{2}$ achieved by electro deposition technique demonstrated an excellent rate capability and cycle life, showing no signs of electrode degradation. The one-step, simple and scalable synthesis protocol developed here makes it viable for integrating into current energy industry and can prove critical in applications such as thin film batteries and micro devices.

\section{Author contributions}

The manuscript was written through contributions of all authors. All authors have given approval to the final version of the manuscript.

\section{Funding sources}

A. G. acknowledges financial support from Netherlands organization for scientific research (NWO) under the framework of Rubicon program (project number 680-50-1205).

\section{References}

1 L. S. Panchakarla, K. S. Subrahmanyam, S. K. Saha, A. Govindaraj, H. R. Krishnamurthy, U. V. Waghmare and C. N. R. Rao, Synthesis, Structure, and Properties of Boronand Nitrogen-Doped Graphene, Adv. Mater., 2009, 21(46), 4726-4730.

2 L. Ci, L. Song, C. Jin, D. Jariwala, D. Wu, Y. Li, A. Srivastava, Z. F. Wang, K. Storr, L. Balicas, et al., Atomic Layers of Hybridized Boron Nitride and Graphene Domains, Nat. Mater., 2010, 9, 430-435.

3 A. L. M. Reddy, A. Srivastava, S. R. Gowda, H. Gullapalli, M. Dubey and P. M. Ajayan, Synthesis of Nitrogen-Doped Graphene Films for Lithium Battery Application, ACS Nano, 2010, 4, 6337-6342.

4 K. Pi, K. M. McCreary, W. Bao, W. Han, Y. F. Chiang, Y. Li, S.-W. Tsai, C. N. Lau and R. K. Kawakami, Electronic Doping and Scattering by Transition Metals on Graphene, Phys. Rev. B: Condens. Matter Mater. Phys., 2009, 80, 75406.

5 R. Rao, G. Chen, L. M. R. Arava, K. Kalaga, M. Ishigami, T. F. Heinz, P. M. Ajayan and A. R. Harutyunyan, Graphene as an Atomically Thin Interface for Growth of Vertically Aligned Carbon Nanotubes, Sci. Rep., 2013, 3, 1891.

6 Z. Yan, L. Ma, Y. Zhu, I. Lahiri, M. G. Hahm, Z. Liu, S. Yang, C. Xiang, W. Lu, Z. Peng, et al., Three-Dimensional MetalGraphene-Nanotube Multifunctional Hybrid Materials, ACS Nano, 2013, 7, 58-64.

7 Z. Chen, W. Ren, L. Gao, B. Liu, S. Pei and H.-M. Cheng, Three-Dimensional Flexible and Conductive Interconnected Graphene Networks Grown by Chemical Vapour Deposition, Nat. Mater., 2011, 10, 424-428.

8 Y. Hernandez, V. Nicolosi, M. Lotya, F. M. Blighe, Z. Sun, S. De, I. T. McGovern, B. Holland, M. Byrne, Y. K. Gun'Ko, et al., High-Yield Production of Graphene by Liquid-Phase Exfoliation of Graphite, Nat. Nanotechnol., 2008, 3, 563-568.

9 M. J. McAllister, J.-L. Li, D. H. Adamson, H. C. Schniepp, A. A. Abdala, J. Liu, M. Herrera-Alonso, D. L. Milius, R. Car, R. K. Prud'homme, et al., Single Sheet Functionalized 
Graphene by Oxidation and Thermal Expansion of Graphite, Chem. Mater., 2007, 19, 4396-4404.

10 S. Stankovich, D. A. Dikin, R. D. Piner, K. A. Kohlhaas, A. Kleinhammes, Y. Jia, Y. Wu, S. T. Nguyen and R. S. Ruoff, Synthesis of Graphene-Based Nanosheets via Chemical Reduction of Exfoliated Graphite Oxide, Carbon, 2007, 45, 1558-1565.

11 W. Gao, L. B. Alemany, L. Ci and P. M. Ajayan, New Insights into the Structure and Reduction of Graphite Oxide, Nat. Chem., 2009, 1, 403-408.

12 K. S. Kim, Y. Zhao, H. Jang, S. Y. Lee, J. M. Kim, K. S. Kim, J.-H. Ahn, P. Kim, J.-Y. Choi and B. H. Hong, Large-Scale Pattern Growth of Graphene Films for Stretchable Transparent Electrodes, Nature, 2009, 457, 706-710.

13 A. Srivastava, C. Galande, L. Ci, L. Song, C. Rai, D. Jariwala, K. F. Kelly and P. M. Ajayan, Novel Liquid Precursor-Based Facile Synthesis of Large-Area Continuous, Single, and Few-Layer Graphene Films, Chem. Mater., 2010, 22, 34573461.

14 X. Li, W. Cai, L. Colombo and R. S. Ruoff, Evolution of Graphene Growth on $\mathrm{Ni}$ and $\mathrm{Cu}$ by Carbon Isotope Labeling, Nano Lett., 2009, 9, 4268-4272.

15 H. Gullapalli, A. L. M. Reddy, S. Kilpatrick, M. Dubey and P. M. Ajayan, Graphene Growth via Carburization of Stainless Steel and Application in Energy Storage, Small, 2011, 7, 1697-1700.

16 P. Lian, X. Zhu, S. Liang, Z. Li, W. Yang and H. Wang, Large Reversible Capacity of High Quality Graphene Sheets as an Anode Material for Lithium-Ion Batteries, Electrochim. Acta, 2010, 55, 3909-3914.

17 E. Yoo, J. Kim, E. Hosono, H. Zhou, T. Kudo and I. Honma, Large Reversible Li Storage of Graphene Nanosheet Families for Use in Rechargeable Lithium Ion Batteries, Nano Lett., 2008, 8, 2277-2282.

18 L. Wang, X. He, J. Li, J. Gao, M. Fang, G. Tian, J. Wang and S. Fan, Graphene-Coated Plastic Film as Current Collector for Lithium/sulfur Batteries, J. Power Sources, 2013, 239, 623-627.

19 S. J. Richard Prabakar, Y.-H. Hwang, E. G. Bae, D. K. Lee and M. Pyo, Graphene Oxide as a Corrosion Inhibitor for the Aluminum Current Collector in Lithium Ion Batteries, Carbon, 2013, 52, 128-136.

20 J. Ning, L. Hao, X. Zhang, M. Liang and L. Zhi, High-Quality Graphene Grown Directly on Stainless Steel Meshes through CVD Process for Enhanced Current Collectors of Supercapacitors, Sci. China: Technol. Sci., 2014, 57, 259-263.

21 Y. Hu, X. Li, J. Wang, R. Li and X. Sun, Free-Standing Graphene-carbon Nanotube Hybrid Papers Used as Current Collector and Binder Free Anodes for Lithium Ion Batteries, J. Power Sources, 2013, 237, 41-46.

22 L. Li, A.-R. O. Raji and J. M. Tour, Graphene-Wrapped $\mathrm{MnO}_{2}-$ Graphene Nanoribbons as Anode Materials for High-Performance Lithium Ion Batteries, Adv. Mater., 2013, 25, 6298-6302.

23 A. Yu, H. W. Park, A. Davies, D. C. Higgins, Z. Chen and X. Xiao, Free-Standing Layer-By-Layer Hybrid Thin Film of
Graphene- $\mathrm{MnO}_{2}$ Nanotube as Anode for Lithium Ion Batteries, J. Phys. Chem. Lett., 2011, 2, 1855-1860.

24 X. Cao, Y. Shi, W. Shi, X. Rui, Q. Yan, J. Kong and H. Zhang, Preparation of $\mathrm{MoS}_{2}$-Coated Three-Dimensional Graphene Networks for High-Performance Anode Material in Lithium-Ion Batteries, Small, 2013, 9, 3433-3438.

25 C. Zhu, X. Mu, P. A. van Aken, Y. Yu and J. Maier, SingleLayered Ultrasmall Nanoplates of $\mathrm{MoS}_{2}$ Embedded in Carbon Nanofibers with Excellent Electrochemical Performance for Lithium and Sodium Storage, Angew. Chem., 2014, 126, 2184-2188.

26 U. K. Sen and S. Mitra, High-Rate and High-Energy-Density Lithium-Ion Battery Anode Containing 2D $\mathrm{MoS}_{2}$ Nanowall and Cellulose Binder, ACS Appl. Mater. Interfaces, 2013, 5, 1240-1247.

27 M. Wang, G. Li, H. Xu, Y. Qian and J. Yang, Enhanced Lithium Storage Performances of Hierarchical Hollow $\mathrm{MoS}_{2}$ Nanoparticles Assembled from Nanosheets, ACS Appl. Mater. Interfaces, 2013, 5, 1003-1008.

28 T. Stephenson, Z. Li, B. Olsen and D. Mitlin, Lithium Ion Battery Applications of Molybdenum Disulfide $\left(\mathrm{MoS}_{2}\right)$ Nanocomposites, Energy Environ. Sci., 2013, 7, 209-231.

29 X. Xiong, W. Luo, X. Hu, C. Chen, L. Qie, D. Hou and Y. Huang, Flexible Membranes of $\mathrm{MoS}_{2} / \mathrm{C}$ Nanofibers by Electrospinning as Binder-Free Anodes for HighPerformance Sodium-Ion Batteries, Sci. Rep., 2015, 5, 9254.

30 H. Li, K. Yu, H. Fu, B. Guo, X. Lei and Z. Zhu, $\mathrm{MoS}_{2} /$ Graphene Hybrid Nanoflowers with Enhanced Electrochemical Performances as Anode for Lithium-Ion Batteries, J. Phys. Chem. C, 2015, 119, 7959-7968.

31 A. W. Maijenburg, M. Regis, A. N. Hattori, H. Tanaka, K.-S. Choi and J. E. ten Elshof, $\mathrm{MoS}_{2}$ Nanocube Structures as Catalysts for Electrochemical $\mathrm{H}_{2}$ Evolution from Acidic Aqueous Solutions, ACS Appl. Mater. Interfaces, 2014, 6, 2003-2010.

32 J. Díaz, G. Paolicelli, S. Ferrer and F. Comin, Separation of the $\mathrm{sp}^{3}$ and $\mathrm{sp}^{2}$ components in the $\mathrm{C} 1 \mathrm{~s}$ photoemission spectra of amorphous carbon films, Phys. Rev. B: Condens. Matter Mater. Phys., 1996, 54, 8064-8069.

33 I. Palchan, M. Crespin, H. Estrade-Szwarckopf and B. Rousseau, Graphite Fluorides: An XPS Study of a New Type of $\mathrm{C}=$ F Bonding, Chem. Phys. Lett., 1989, 157, 321-327.

$34 \mathrm{H}$. Hwang, H. Kim and J. Cho, $\mathrm{MoS}_{2}$ Nanoplates Consisting of Disordered Graphene-like Layers for High Rate Lithium Battery Anode Materials, Nano Lett., 2011, 11, 4826-4830.

35 J. Xiao, D. Choi, L. Cosimbescu, P. Koech, J. Liu and J. P. Lemmon, Exfoliated $\mathrm{MoS}_{2}$ Nanocomposite as an Anode Material for Lithium Ion Batteries, Chem. Mater., 2010, 22, 4522-4524.

36 J. Wang, J. Liu, D. Chao, J. Yan, J. Lin and Z. Shen, SelfAssembly of Honeycomb-like $\mathrm{MoS}_{2}$ Nanoarchitectures Anchored into Graphene Foam for Enhanced Lithium-Ion Storage, Adv. Mater., 2014, 26, 7162-7169.

37 J. Wang, J. Liu, J. Luo, P. Liang, D. Chao, L. Lai, J. Lin and Z. Shen, $\mathrm{MoS}_{2}$ architectures supported on graphene foam/ carbon nanotube hybrid films: highly integrated frameworks with ideal contact for superior lithium storage, J. Mater. Chem. A, 2015, 3, 17534-17543. 